\title{
Chronic Neutrophilic Leukemia: A Rare Chronic Myeloproliferative Disorder
}

\author{
Amar RANJAN, Raja PRAMANIK, Ritu GUPTA, Pranay TANWAR, Sachidanandjee BHARATI, \\ Mukurdipi RAY, Rajkumar CHANDOKE, Ashutosh KUMAR
}

Dr. BRA-IRCH, All India Institute of Medical Sciences, Laboratory Oncology Unit, New Delhi, INDIA

To the Editor,

Chronic Neutrophilic leukemia (CNL) is rare chronic myeloproliferative disorder (CMPD). Only 150 cases have been reported till date. It presents with neutrophilia, hypercellular bone marrow and hepatosplenomegaly. Philadelphia (Ph) chromosome is negative. Diagnosis requires exclusion of reactive neutrophilia and other Myeloproliferative neoplasms (MPN). Here a case is being presented who was 64 year old male complained abdominal pain after meals for one year. On clinical examination there was hepatosplenomegaly. There was history of several surgical interventions in last 15 years. The patient was operated for appendicitis 5 yrs back. . There was right sided pneumothorax 7 year back, for which intercostal tube was put. Again operation for anorectal fistula was done 15 years back. No history of tuberculosis, diabetes or hypertension was found. Serum uric acid level was $12.10 \mathrm{mg} / \mathrm{dl}$ (normal: $2.0-7.5 \mathrm{mg} / \mathrm{dl})$.

Peripheral blood smear (PBS) revealed total leukocyte count $34000 / \mathrm{cmm}$ with neutrophilia (>90\%). Hypersegmented \& hypergranular neutrophils were abundant without left shift (Figure 1). Haemoglobin was $14 \mathrm{gm} /$ $\mathrm{dl}$, platelet count was $<40.000 / \mathrm{cmm}$.

Bone Marrow Aspirate (BMA) showed hemopoietic cells of all series with ME ratio 10:1. There was focal clustering of plasma cells although of benign nature, raising suspicion of plasma cell myeloma (Figure 2). No dysplastic change was noted in any cell lineage (Figure 2 and 3). Leucocyte Alkaline Phosphatase (LAP) score was markedly high. Bone Marrow Biopsy confirmed the findings of aspirate.

Fluorescent in situ hybridization (FISH) was done for $\operatorname{del}(5 q)$, del (7q) \& del (20q), all were negative.

Real Time Quantitative Polymerase Chain Reaction (RTQPCR) for bcr-abl translocation assay was done. It showed p190, p210 \& p230 negative. PCR and Gel Electrophoresis for jak-2 mutation $\mathrm{V} 617 \mathrm{~F}$ was also negative.

Studies for plasma cell myeloma like urinary \& serum protein electrophoresis with skeletal survey were negative. After ruling out other causes of neutrophilia, CNL was finally considered.

Patient was started treatment with Hydroxyurea $500 \mathrm{mg}$ TDS \& Allopurinol 100mg TDS with oral hydration. Following two months improvement seen with Platelet count 53000/cmm \& Hb $14 \mathrm{gm} / \mathrm{dl}$. To date, he is on monotherapy with hydroxyurea with adequate suppression of leukocyte count.

MPN is a clonal hematopoietic stem cell disorders characterized by proliferation of one or more elements of myeloid lineage. In this case, marked leukocytosis consisting primarily of mature neutrophils was at first suspicious for MPN/CML. But there was absence of basophilia, Philadelphia chromosome and BCR-ABL1 fusion transcript. It excluded CML. Negativity of JAK2 mutation excluded other MPN such as polycythemia vera, essential thrombocythemia and primary myelofibrosis. Now the focus was on either CNL or atypical CML, BCR-ABL1 negative. 


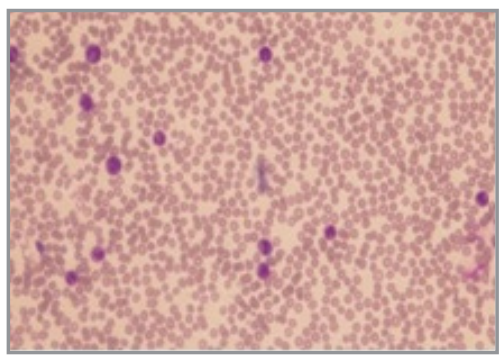

Figure 1. Peripheral blood smear, May Grunwald Giemsa stain,20x

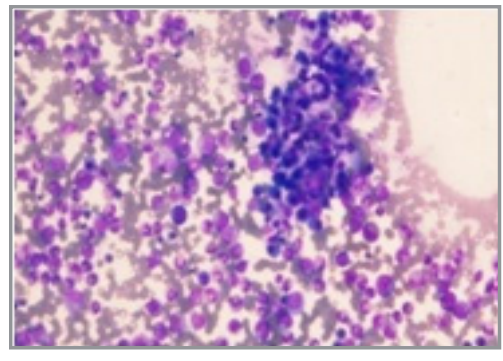

Figure 2. Bone Marrow Aspirate, May Grunwald Giemsa stain, 40x, plasma cell (dark coloured) cluster

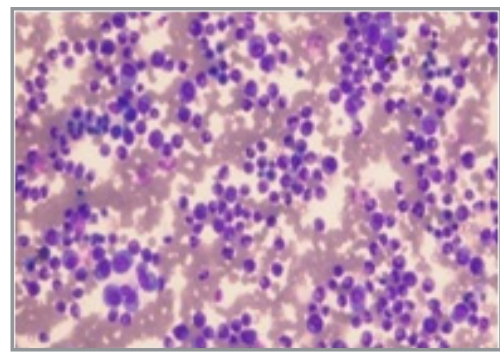

Figure 3. Bone Marrow Aspirate, May Grunwald Giemsa stain,20x
BCR-ABL1 fusion protein, $\mathrm{p} 230$ was negative, which excluded atypical CML.

As per current World Health Organization (WHO) criteria, a diagnosis of CNL is more appropriate in this case. First, peripheral blood and bone marrow show a predominance of mature myeloid forms without significant dysgranulopoiesis. Second, neutrophil precursors make up less than $10 \%$ of circulating leukocytes.

CNL has attained the formal recognition as a distinct entity in Revised WHO classification of Myeloid Neoplasm. It presents in elderly adults with an equal distribution between male and female. ${ }^{1,2}$ Clinical presentations are variable. It involves peripheral blood, bone marrow, spleen, liver commonly, but can involve any tissue. ${ }^{1}$

The patient may have history of bleeding from mucocutaneous surface or GIT. Our patient was having erosive antral gastritis \& duodenitis, which may be early sign for bleeding. Presence of high uric acid level $12.10 \mathrm{mg} / \mathrm{dl}$ (normal: $2-7.5 \mathrm{mg} / \mathrm{dl}$ ) also favoured the diagnosis of CNL in this case. In $10 \%$ of cases, cytogenetic abnormalities of $+8,+9,+21$, del(20q), $\operatorname{del}(11 q) \& \operatorname{del}(12 p)$ are seen. ${ }^{1}$ If BCR-ABL1 fusion protein, p230 is found, atypical CML is diagnosed. This case was negative for $\mathrm{p} 190, \mathrm{p} 210 \& \mathrm{p} 230$. Occasional patient with JAK2 mutation has been reported in CNL, but this case was negative. Multiple surgical interventions for the last 15 years was probably not associated with development of CNL. A Elliott et al 2005 studied 12 cases of CNL. None of the patients was having any significant co-morbidity, such as infection, malignancy or systemic inflammatory disease, capable of causing a reactive neutrophilia or leukemoid reaction. ${ }^{2}$

In $20 \%$ of reported cases, neutrophilia was associated with plasma cell dyscrasia. To date no CNL has been reported with Multiple Myeloma (MM), in which the evidence of clonality by molecular technique has been convincingly demonstrated in neutrophils. In $\mathrm{MM}$, neutrophilia may result from CK release from abnormal plasma cells. Evolution to AML has been seen in one case of CNL with MM. ${ }^{1}$

The clinical course of CNL is heterogeneous. Disease acceleration often manifests with progressive neutrophilia with resistance to previously effective therapy, progressive splenomegaly or worsening thrombocytopenia (not related to therapy), or with cytogenetic clonal evolution. Blast Transformation (exclusively myeloid to date), occurs in significant proportion of cases at a median duration of 21 months (range 3-94 months) from the time of diagnosis. No hematologic complete remission has been described to date following induction therapy for accelerated or blast phase in CNL. ${ }^{3}$

Optimal therapy remains to be defined. Splenectomy cannot be recommended for fear of worsening neutrophilia. Treatment to date is cytoreductive agents such as hydroxyurea. Approximately $75 \%$ have a clinical response in terms of decrease in leukocytosis and/or splenomegaly. Clinical responses are maintained for a median of 12 months (range 1 - 87 months). Allogenic sibling Stem Cell Transplantation has now been reported in five cases (ages 15, 40, 44, 49, and 60 years) in the chronic or accelerated phase or following induction chemotherapy for blast transformation. The median survival was 23.5 months (range: 1 - 106). Causes of death included intracranial haemorrhage, progressive disease, blast transformation, and infection. ${ }^{4}$

Thus the diagnosis of CNL needs a careful exclusion of different clinical entities with similar clinical picture by detection of molecular abnormalities. This facility is not available at many centres in develop- 
Table 1. Diagnostic criteria for chronic neutrophilic leukaemia

1. Peripheral blood leukocytosis, WB $\geq 25 \times 10^{9} / \mathrm{L}$

Segmented neutrophils and band forms are $>80 \%$ of blood cells

Immature granulocytes (promyelocytes, myelocytes, metamyelocytes) $<10 \%$ of white blood cells

Myeloblasts $<1 \%$ of white blood cells

2. Hypercellular bone marrow biopsy

Neutrophilic granulocytes increased in percentage and number

Myeloblasts $<5 \%$ of nucleated marrow cells

Neutrophilic maturation pattern normal

Megakaryocytes normal or left shifted

3. Hepatomegali

4. No identifiable cause for physiologic neutrophilia or, if present, demonstration of clonality of myeloid cells by cytogenetic or molecular studies

No infectious or inflammatory process

No underlying tumour

5. No Philadelphia chromosome or BCR-ABL1 fusion gene

6. No rearrangement of PDGFRA, PDGFRB or FGFR1

7. No evidence of polycythaemia vera, primary myelofibrosis or essential thrombocythaemia

8. No evidence of a myelodysplastic syndrome or a myelodysplastic/myeloproliferative neoplasm

No granulocytic dysplasia

No myelodysplastic changes in other myeloid lineages

Monocytes $<1 \times 10^{\circ} / L$

ing countries. But the characterization of molecular profiles of CNL and other rare MPN would aid not only in its classification and diagnosis, but could also yield novel treatment options. Further study is needed over this.

\section{REFERENCES}

1. Bain BJ, Brunning RD, Vardiman JW, Thiele J. Chronic neutrophilic leukemia. In: WHO classification of tumors of hematopoietic and lymphoid tissue. Steven HS, Elias C, Nancy LH, et al. 4th edition, International Agency for research on Cancer, Lyon, 2008: 38-39.

2. Elliott A, Hanson CA, Dewald GW, et al. WHO-defined chronic neutrophilic leukemia: a long-term analysis of 12 cases and a critical review of the literature. Leukemia 19: 313-317, 2005.

3. Jason G, Julia EM, Tracy IG, Jeffrey WT. The new genetics of chronic neutrophilic leukemia and atypical. Blood 122: 1701-11, 2013.

4. Elliott MA, Dewald GW, Tefferi A, Hanson CA. Chronic neutrophilic leukemia (CNL): a clinical, pathologic and cytogenetic study, Leukemia 15: 35-40, 2001.

\section{Correspondence}

Dr. Rajkumar CHANDOKE

Department of Pathology

Employee State Insurance Hospital

Basai darapur

New Delhi / INDIA

Tel: $91-9811348034$

e-mail: drchandoke@gmail.com 